\title{
LXXIV. The action of ultra-violet light on chlorine
}

\section{E.B. Ludlam D.Sc.}

To cite this article: E.B. Ludlam D.Sc. (1912) LXXIV. The action of ultra-violet light on chlorine, Philosophical Magazine Series 6, 23:137, 757-772, DOI: 10.1080/14786440508637274

To link to this article: http://dx.doi.org/10.1080/14786440508637274

曲 Published online: 08 Jun 2010.

Submit your article to this journal $₫$

Q View related articles $₫$

4 Citing articles: 4 View citing articles 준 
The superiority of the above method will be realized when it is compared with the proofs of the equivalent relations by the authors named.

In the application of the second law to mixtures similar transformations of the differential equation are often useful. In that case the equation has to be supplemented by terms containing quantities which determine the amounts of the various components of the mixture. These terms may be of the form $\mu d m$, equal in number to the number $p$ of the components, where $m$ stands for the quantity (e.g. mass or number of gramme-molecules) of each component, and $\mu$ is called the potential (Gibbs). In many cases it is more appropriate to refer the equation to a definite quantity (e.g. unit mass or one molecule), and introduce variables which determine the composition of the mixture, e.g. the ratios $x, y, \ldots$ of the amounts of the $2 \mathrm{nd}, 3 \mathrm{rd}, \ldots$ component to the whole ; the equation then assumes the form

$$
d u=\mathrm{T} d s+\stackrel{n}{\Sigma} \mathrm{A} d \alpha+\sum^{p-1} \mathrm{X} d x .
$$

The total number of forms into which this equation may be put by the subtraction of expressions like $d(\mathrm{~T} / s), d(\mathrm{~A} \alpha)$, or $d(\mathrm{X} x)$, is obviously $2^{n+p}$, giving rise to the introduction of the same number of thermodynamical functions (including $u$ itself) and allowing the deduction of $(n+p)(n+p-1) 2^{n+p-1}$ thermodynamical relations of the special simple form under consideration. A further discussion of these relations or of the application of the functions $e . g$. for the construction of thermodynamical surfaces would be out of place here *.

\section{The Action of Ultra-violet Light on Chlorine. $B y$ E. B. Ludlam, D.Sc. $\dagger$}

THE action of ultra-violet light upon many elements 1 causes them to eject electrons, and the more electropositive the element the more easily is the electron ejected. 'Thus, the alkali metals are the most sensitive to ultra-violet light and less positive metals less so; nevertheless, an element so electronegative as oxygen gas appears to be ionized by ultra-violet light, presumably by the detachment of an electron, as in the case of metals. It was therefore a matter of interest to ascertain whether chlorine, the most

* Compare H. Kamerlingh Onnes and W. H. Keesom, Encyklopïäie der Math. Wiss. v., which is to appear shortly.

+ Communicated by the Author. 
electronegative element upon which we can experiment, showed any similar photoelectric effect.

Lenard has found that in the case of oxygen a shorter wave-length is necessary to produce ionization than is required to convert the oxygen into ozone. Shenstono carried out an experiment ${ }^{*}$ to discover if any ozone-like modification of chlorine was produced by means of the silent electric discharge, but his result was purely negative.

Chlorine, being green, absorbs light in the visible portion of the spectrum, and there is no doubt that light of this wave-length is effective in producing chemical change. It was, therefore, of interest to ascertain whether any ionization of the gas was measurable when it wos exposed to light of various wave-lengths, including such light as is effective in ionizing oxygen. In this connexion it is worthy of note that the spectrum of chlorine in the ultra-violet region does not consist of lines but of a band, and in this region the emission spectrum is not the same as the absorption spectrum; from this it may be anticipated that the mechanism of the absorption in the ultra-violet band is not the same as in the portion consisting of lines, and this band absorption might fully prevent the ejection of electrons.

But, of all the problems connected with chlorine, the most fascinating is the mechanism of its union with hydrogen under the influence of light. Under suitable conditions the union talses place with explosion, but, that this may occur, the gases must be mixed in equal volumes, they must be free from certain impurities which insede the renction, the illumination must be powerful, and the gases must not be absolutely dry.

The actual amount of energy supplied by the light is negligible compared $w$ ith that liberated by the reaction, therefore the light plays the part of the trigger to the explosion, and the new equilibrium which it sets up is not displaced by the energy supplied by the light.

It was mainly with a view to obtain some know]edge of the mechanism of this catalytic trigger action that the work described in this paper was undertaken, for one point of first importance to settle is the question as to whether light is capable of ionizing chlorine.

Before dealing with the actual experiments, it may be serviceable to state briefly some of the most recent results obtained by Lenard and his collaborators, as these have not yet appeared in a publication of wide circulation + .

* Shenstone, J. C. S. Trans. rol. 1xxi. p. 487.

+ See Lenard \& Ramsauer, Trans, Heidelberg Academy, Aug. 2, 1910 to Aug. 4, 1911, fire parts. 
Long ago Lenard showed that the action of ultra-violet light on gases produced three kinds of effects * :-

1. The gas may conduct electricity owing to the production of positive and negative ions.

2. Condensation nuclei may be formed, devoid of electric charge $\dagger$.

3. Chemical molecular change may take place $\left(3 \mathrm{O}_{2}\right.$ to $\left.2 \mathrm{O}_{3}\right)$.

All these effects are due to the action of the periodic electric forces on the molecules of the gas.

Nuclei.-The condensation nuclei persist longer than the ions, from which they can be separated by means of a powerful electric field; they are unaffected by cooling to $-70^{\circ} \mathrm{C}$., but destroyed at $180^{\circ} \mathrm{C}$.; their diameter appears to be some 25 times that of the molecule of the gas, but varies with the duration of illumination, increasing with the time; their formation was at first thought to be analogous to the production of ozone, but is more probably due to secondary chemical reactions (e. g., $\mathrm{NH}_{3}$ oxidized to $\mathrm{NH}_{4} \mathrm{NO}_{2}$, \&c.). When the gases, not specially purified by cooling, are exposed to short wave-lengths (below $1.80 \mu \mu$ ), there is no diminution in the number of condensation nuclei (as judged by means of a steam jet) whether the ions are removed by a powerful electric field or not. Hence the nuclei are more effective than ions in bringing about the condensation of supersaturated steam.

When, however, the gases are highly purified, most effectively by freezing out impurities by means of liquid air, no nuclei are formed, proving that they are due to minute traces of vapours present in the air ; the effect of the merest traces of water vapour, ammonia, or vapour from rubber tubing is most striking.

Ions.-The production of ions of molecular dimensions is explained by supposing the loss of a negative electron by one molecule and the gain of it by another. Larger ions could arise by agglomeration, but this process does not go far, or by the attachment of a small ion to a larger particle (nucleus) suspended in the gas.

With gases not highly purified, the longer and more powerful the illumination the larger the ions, but with gases purified by cooling to $-78^{\circ} \mathrm{C}$. only ions of molecular size are formed; hence, it appears that large ions are due to the acquisition of an electron by a nucleus.

* Lenard \& Wolf, Ann. d. Phys. u. Chem. vol. iii. F (1889); Ann. d. Phys. vols. i. \& iii. (1900).

$t$ See also C. T. R. Wilson, Phil. Trans, rol. cxcii. (1899). 
In this way the nuclei have the effect of conserving the ions, damping their velocity and so preventing their rapid recombination and diffusion to the walls *.

Ozone.-Is produced even in air which has been purified by cold and must be a consequence of the direct action of the light on the molecules of oxygen; its production is caused by wave-lengths longer than $200 \mu \mu$, and is not associated with any photoelectric effect.

\section{Effect on the Walls of the Vessel.}

All the experiments on ionization of gases are complicated by the action of the light on the walls of the containing vessel. No conductor is available for the walls which does not either give out ions itself, or contaminate the gases. The wave-length at which the wall-effect begins is longer than that at which the gas effect first takes place ; further, the ions produced are always negative, whereas the gas effect produces an equal number of positive and negative ions; consequently, the excess of negative ions over positive is throughout these experiments ascribed to the action of light on the walls of the vessel.

Wave-length.-A careful study of the effects observed through different media leads to the conclusion that the difference in properties between visible violet and the adjoining ultra-violet is less than between some portions of the ultra-violet region, and this may account for numerous discrepancies between the results of different observers who have unwittingly been experimenting with light of different wave-lengths.

\section{Experimental.}

The source of light employed was a spark of about one centimetre in length produced between aluminium terminals connected to a coil of special construction, wound with a secondary of low resistance and provided with a condenser of great capacity. The coil was supplied with a current of 20 amperes broken by a Wehnelt interrupter. The coil and spark-gap were both enclosed in tin boxes connected to earth.

Chlorine is a difficult gas to work with. Accordingly, the plan followed was to commence with air, to observe the effects already established with regard to it and proceed to examine the effect of an increasing proportion of chlorine, until pure chlorine itself was under investigation; then, to add intentionally certain vapours and note their effect.

* The reverse action, destruction of ions, has been observed in cases where the concentration of the nuclei was very great. 
Table of Wave-lengths in the Ultra-violet Region.

\begin{tabular}{|c|c|c|c|}
\hline $\begin{array}{c}\text { Wave-length. } \\
\mu \mu\end{array}$ & & $\begin{array}{l}\text { Deecription } \\
\text { and } \\
\text { abbrevintion. }\end{array}$ & Remarks. \\
\hline $440-380$ & & Violet. & Visible. \\
\hline $380-340$ & & $\begin{array}{c}\text { Glass } \\
\text { Ultra-violet } \\
\text { (Glass-violet). }\end{array}$ & $\begin{array}{l}\text { Passes through ordinary glass } \\
\text { (in fairly thin layers); Carbon } \\
\text { arc rich source. }\end{array}$ \\
\hline $340-300$ & $\stackrel{0}{\frac{0}{0}}$ & $\begin{array}{c}\text { Jena glass } \\
\text { Ultra-violet } \\
\text { (Jena-glass * } \\
\text { violet). }\end{array}$ & $\begin{array}{l}\text { Passes thirough Jena ultra-violet } \\
\text { crown glass: strongly emitted } \\
\text { by the Uviol Mercury lamp. }\end{array}$ \\
\hline $300-2=0$ & 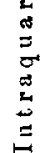 & $\begin{array}{c}\text { Qunrtz-glass } \\
\text { ['ltri-violet } \\
\text { (Quartz-glass* } \\
\text { viole1). }\end{array}$ & $\begin{array}{l}\text { Passes through fused silica (and } \\
\text { calc-spar) in fairiy thin layers: } \\
\text { list portion of strong radiation } \\
\text { from the Quartz Mercury and } \\
\text { Amalgam lanps. }\end{array}$ \\
\hline $2: 0-180$ & & $\begin{array}{l}\text { Quartz-crystal } \\
\text { Cltra-violet } \\
\text { (Crystal-violet). }\end{array}$ & $\begin{array}{l}\text { Passes through crystallized quartz, } \\
\text { gypsum, rock-salt in fairly thin } \\
\text { layers: separable in the quartz- } \\
\text { spectrograph. }\end{array}$ \\
\hline $180-120$ & $\frac{2}{0}$ & $\begin{array}{l}\text { Fluorspar } \\
\text { Eltra-viulet } \\
\text { (Schuntan- } \\
\text { violet). }\end{array}$ & $\begin{array}{l}\text { Passes through good fluorspar, } \\
\text { but completely absorbed by a } \\
\text { short space of uir: separable in } \\
\text { a vacuum-fluorspar-spectro- } \\
\text { graph. }\end{array}$ \\
\hline $120-30(?)$ & \begin{tabular}{l}
2 \\
\multirow{2}{*}{} \\
0 \\
0 \\
$=$ \\
0
\end{tabular} & $\begin{array}{l}\text { Reflex vlitra- } \\
\text { riolet } \\
\text { (Reflex violet). }\end{array}$ & $\begin{array}{l}\text { On the dispersion theory strongly } \\
\text { reflected by the abore media: } \\
\text { separable by means of a reflexion } \\
\text { grating. }\end{array}$ \\
\hline Shorter waves & 5 & $\begin{array}{c}\text { Trnns-reflex } \\
\text { Viulet. }\end{array}$ & \\
\hline
\end{tabular}

* The:e two rariati'ns are by no means sharply separated by the absorption of fused quartz, this absorption increasing very gradually in the regiun of $220 \mu \mu$; nevertheless, it may be convenient to reserve separate names for the two portions of quartz-violet.

In early experiments the chlorine was obtained respectively from chlorine water, bleaching powder and sulphuric acid and by the electrolysis of cupric chloride solution, but finally the gas was obtained from liquid chlorine in a steel cylinder.

The gas was dried by passage first over calcium chloride, then over phosphorus pentoxide on broken glass, and finally 
through a filter of purified asbestos in a Jena-glass tube which could be rendered absolutely free from moisture by heating in the blowpipe to a bright red heat.

\section{Diagram of Connexions.}

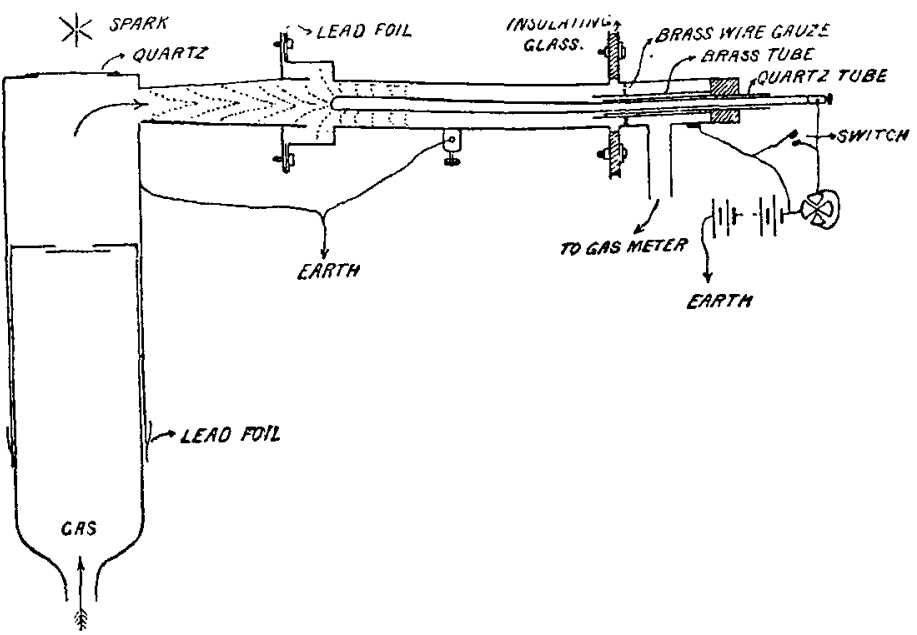

The gas then passed into the brass illuminating vessel, of which the walls, in the later experiments, were gold-plated. It was led in front of a window of crystalline quartz, $\frac{1}{4} \mathrm{~mm}$. thick, and thence into a cylindrical condenser of the type described by Becker*. 'The illumination vessel and the outer cylinder of the condenser were earthed, the axial rod of the condenser was connected by protected wires to the two quadrants of an electrometer, whose sensitiveness was arranged so that one volt gave a deflexion of $300 \mathrm{~mm}$. on the scale. The electrometer case and the metal box in which it was placed were connected with one pole of a ligh-potential battery whose other pole was earthed. The small battery used for charging the needle, and the high-potential battery, were also both enclosed in earthed metal boxes to prevent possible disturbances arising from the sparking in the neighbourhood.

In the earlier experiments the condenser was made entirely of brass, the insulating parts being of special glass, but later brass which had been gold plated was used, and in the concluding experiments both the cylinder and inner rod were of carbon, insulated by means of fused quartz.

From the condenser the gas passed into a gas meter of

* Zeitschr. für Instrumentenkunde, rol, xxix. p. 258 (1909). 
very simple construction and thence into a larga carboy containing slaked lime, and from this a way into the open air. An electric fan was employed to maintain a strong draught and remove such cblorine as might leak into the air.

The series of curves show the kind of result obtained :-

\section{Experiments on Air.}

Curve I.-Represents the results of one of the earliest experiments using ordinary pure dry air. It shows how all the negative ions produced by 1.0 seconds' illumination were caught by a potential difference of two volts, but that with

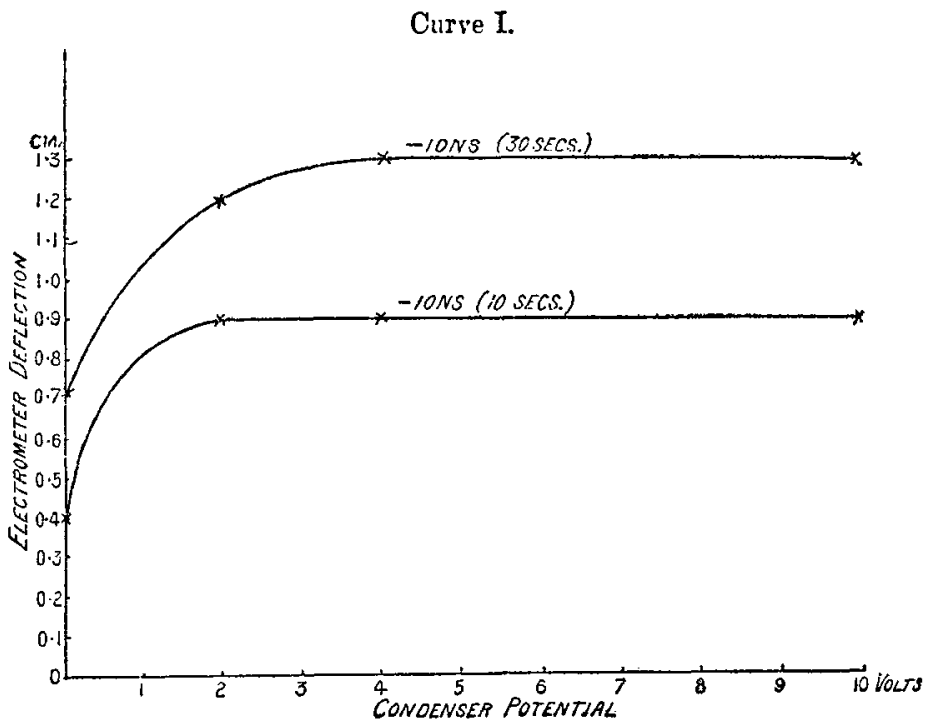

Effect of duration of illumination on the number and mobility of negative ions in air.

three times the illumination larger ions had been formed requiring four volts before the saturation potential was reached, only about 90 per cent. of the ions being caught at 2 volts.

From the formula deduced for his condenser by Becker*, the velocity of the ion corresponding to a saturation potential of two volts works out to 0.28 centimetre per second for one volt per centimetre, and for four volts half that velocity. The formula is, however, only an approximation unless a radial field is secured inside the condenser and the stream of gas is free from eddies, wherens in these experiments such

* A. Becker, Ann. de Phys, vol, xxxi. p. 98 (1910). 
was not the case. The Becker condenser is admirahly suited to the measurement of the mobility of large slow-moving ions, but suffers from the disadvantage that a very perceptible loss of the smaller ions takes place by recombination and diffusion to the walls of the apparatus before the condenser is rearhed. To avoid this the radial field was sacrificed by adopting an unusual system of electrical connexions whereby all the ions of the required sign were drawn towards the axial rod of the condenser which was connected to the electrometer.

Influence of the rate of flow of the gas on the number of ions carried into the condenser.-Compressed air from a cylinder was driven at various speeds through the apparatus and the deflexion due to the negative ions was measured. The number of positive ions was so small in comparison with the negative ions that the latter could be regarded as all produced by the action of the light on the walls, and therefore constant, thns the complication arising from the fact that with increased speed more gas is exposed to the action of the light was avoided. With a speed of 1 litre per minute the deflexion was $1.5 \mathrm{~cm}$., with 3 litres per minute this had increased to $4.2 \mathrm{~cm}$., and to $8 \mathrm{~cm}$. at 5 litres per minute. In consequence of this result a rapid stream of gas was employed in all the later experiments.

\section{Experiments on Chlorine.}

A current of air at a speed of four litres per minute was sent through the apparatus, and chlorine in gradually increasing amounts was mixed with it.

When no chlorine was present very few positive ions were formed, nor was there any marked increase when $0.5,1 \cdot 5$, 15 , and 90 per cent. chlorine were added, the velocity of the mixed gases being always 4 litres per minute.

With the pure chlorine the deflexion was hardly measurable at all (velocity 3 litres per minute, as the meter would not measure more of the heavy gas).

The addition of very small quantities of chlorine (less than -5 per cent.) to pure air was found in some early experiments to diminish the number of negative ions and increase the number of positive ones. The decrease in the negative ions was to be expected, for their number is swelled by the presence of ions from the walls of the vessel, and these are fewer when the light has to traverse a powerfully absorbing gas before striking the walls. 
Influence of a Trace of Carbon Disulplide.

It was found that when the proportion of cllorine was increased beyond about 1 per cent. the number of positive ions began to decrease. It was considered possible that this might be due to increase in the rapidity of recombination accompanying increase in concentration, and accordingly a series of experiments was conducted in which a trace of carbon disulphide was allowed to diffuse into the air containing chlorine, for Lenard had found that this substance, under the influence of ultra-violet light, furnished nuclei capable of conserving the ions.

With air containing only a trace of carbon bisulphide the number of ions produced was only slightly greater than when pure air was employed, but immediately chlorine was added the effect was very marked, a decided increase taking place in the number of positive ions formed.

Curve 11.-Shows a typical example of the results obtained for positive ions in this series of experiments. It will bo

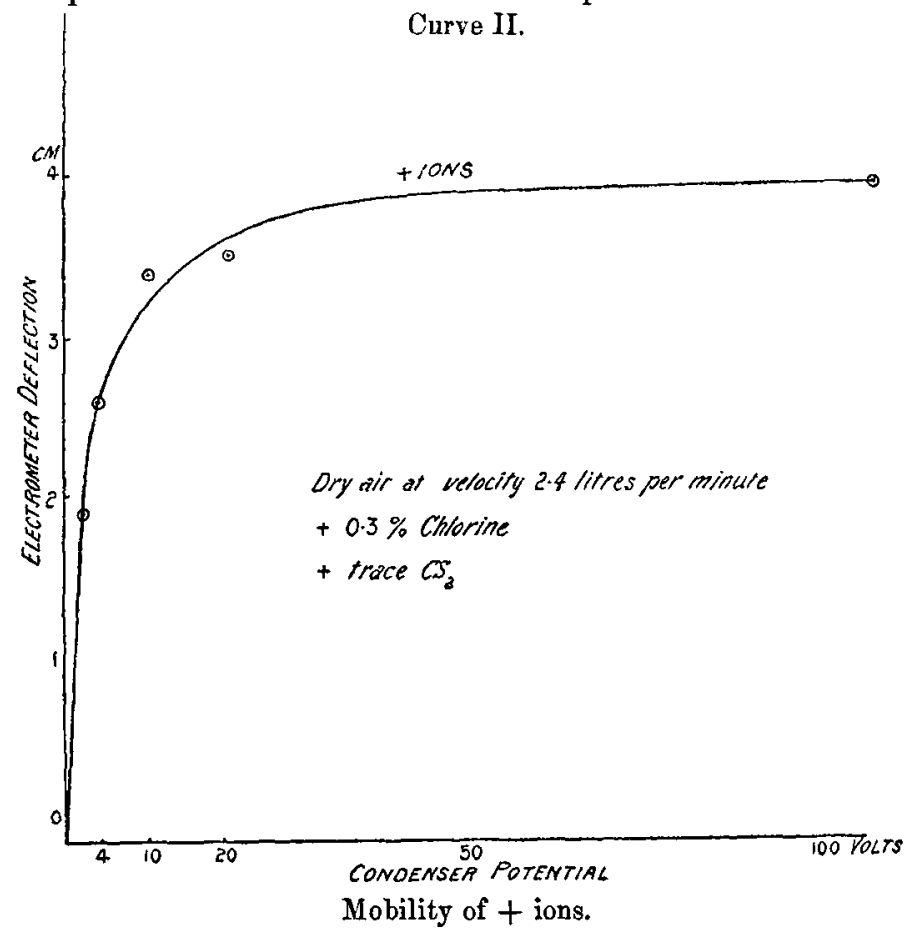

noticed that the curve begins to run horizontally at about 30 volts, signifying that much larger ions are present than in Curve I., moving with less than one tenth the speed. 
Curve III.-The effect of gradually increasing the proportion of chlorine is shown in a striking manner in Curve III. With no chlorine at all, the negative ions are greatly in excess of the positive, but, directly so little as 0.15 per cent. is added, the number drops to one fifth its previous value, and approximates to that of the positive ions.

Curve III.

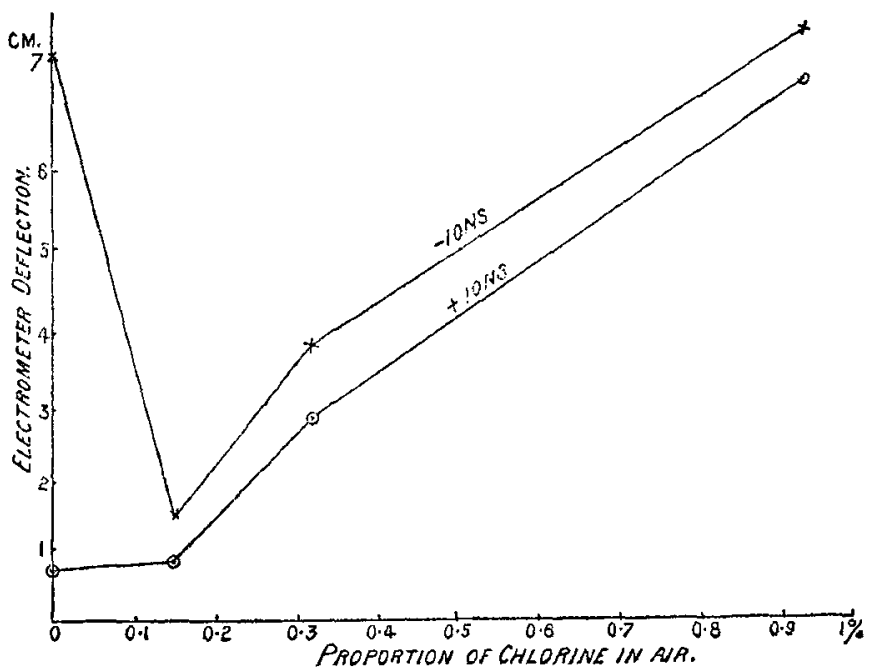

Influenee of small per cent. of chlorine on the ionization of air.

(Potential $=100$ volts.)

With further increase in the quantity of chlorine, both positive and negative ions increase in number side by side, the negatire being always slightly in excess.

In this series of experiments the potential was not varied, being maintained at 100 volts, as previous results had indicated that 100 volts was more than sufficient to catch all the ions. However, to follow any changes which might have taken place in the size and mobility of the ions due to the addition of chlorine, a series of experiments was carried out of which the results are represented in Curve IV.

Curve IV.-The most striking fact indicated by this curve is the small size of the ions produced in the presence of 1 per cent. of chlorine- the two highest curves may be said to run horizontally from a potential of 4 volts, for $0 \cdot 3$ per cent. ehlorine, 30 volts were necessary to catch all the positive ions-a value in agreement with Curve II.

The curve also shows that the first effect of a srnall quantity of chlorine $(0.15$ per cent.) is to increase the size of the ions, for with no chlorine present all are caught at 10 rults; with 
0.15 per cent. chlorine 30 volts are necessary. The drop in the number of negative ions on the first addition of chlorine is again very clearly brought out.

Curve IV.

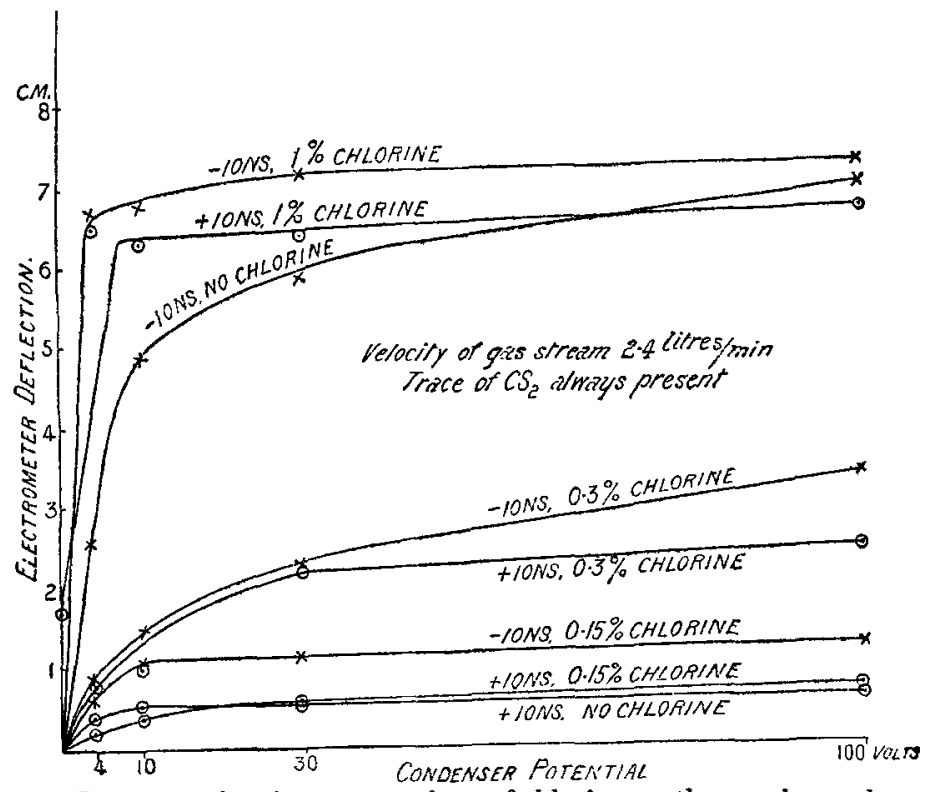

Intluence of various proportions of chlorine on the number and mobility of ions.

Curve $V$ represents the effect observed with increased percentage of chlorine. The number of ions in 3 per cent. chlorine is pretty much the same as was found in air containing only 1 per cent., but when the chlorine is increased to 20 per cent. the ionization drops to about one third the value. This result might have been attributed merely to the increase in the rate of recombination due to the absence of sufficient diluting gas, but another possibility suggested itself, namely, that the stream of cool air drawn past the apparatus by the fan had so lowered the temperature of the carbon bisulphide tube as to reduce its vapour pressure below the minimum necessary to provide a sufficient number of nuclei to efficiently conserve the ions, now that the proportion of chlorine was very considerable. Consequently, the experiment was repeated with the carbon bisulphide tube immersed in water at $27^{\circ} \mathrm{C}$., whereupon the number of ions was almost trebled ${ }^{*}$. This result opened the question as to whether the
*V.P. of $\left.\mathrm{CS}_{2}\right\} 20 \mathrm{~cm}$.
$20^{\circ}$
$2 i^{\circ}$
$30 \mathrm{~cm}$.
$40 \mathrm{~cm}$. npproximately. 
effect observed might not be due to a chemical action between the chlorine and the carbon bisulphide under the influence of the light.

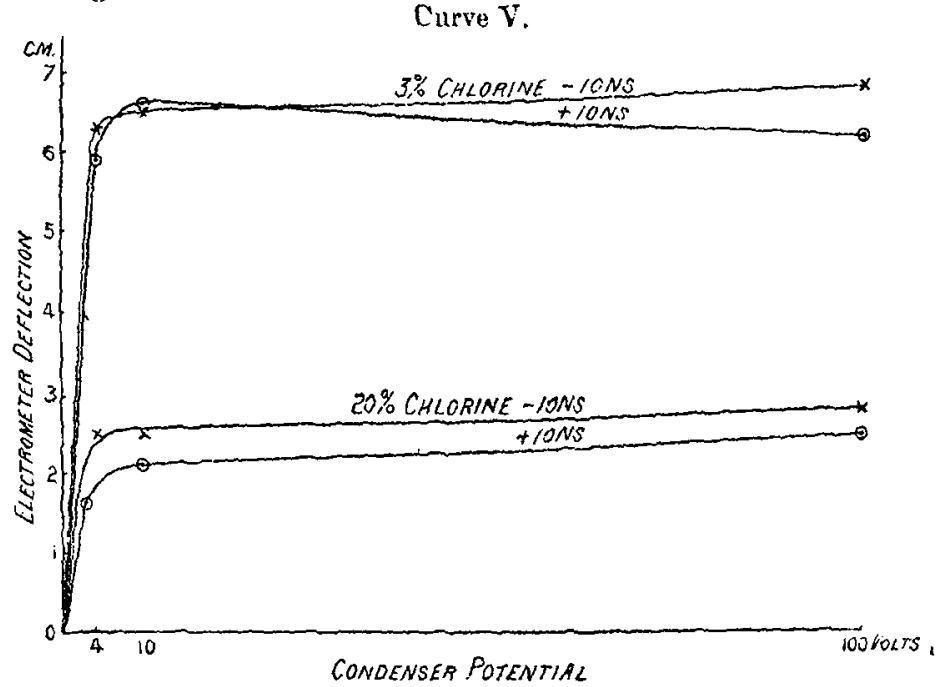

Influence of greater per cent. of chloriue on ions produced in air.

It this were the case, the presence of a large excess of chlorine would result in the absorption of the light by the chlorine without production of ions before the photo-chemical action could come into ylay.

Wave-length.- Throughout ench series of experiments observations were made upon the wave-length of the light producing the various effects, and similar results were always obtained, of which the table below is characteristic.

Air at the rate of 2.4 litres per minute, chlorine 1.5 per cent., trace of carbon bisulphide.

Medium between Spark and Gas.

$3 \mathrm{~cm}$. air, $\frac{2}{4}$ min. quartz

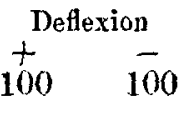
" $\quad 3 \mathrm{~mm}$. gypsum (fine split crystal plate)

$3 \mathrm{~cm}$. air, $3 \mathrm{~mm}$. gypsum (old and rubbed)

$10 \quad 10$

$3 \mathrm{~cm}$. air, calc-spar

$2^{*} \quad 8$

$3 \mathrm{~cm}$. ultra-violet crown glass $\ldots .$. .

Violet crown glass $\ldots \ldots \ldots \ldots \ldots \ldots . . . .$.

44

$\begin{array}{ll}0 & 2\end{array}$

Ordinary glass

* 'This is an unusually low value, 8 is nearer the areraye, although the positive deflexion was usually rather less than the negative when culcepar was used. 
The plate of gypsum, examined in the quartz spectrograph, was still very transparent to wave-lengths of $185 \mu \mu$; the calc-spar had a sharp boundary, being transparent to wavelength $206 \mu \mu$, but opaque to $202 \mu \mu$; the ultra-violet crown glass absorbed all shorter than $290 \mu \mu$. The evidence is sufficient to warrant the conclusion that, for any considerable ionization, wave-lengths shorter than $200 \mu \mu$ are required, and if any ionization is caused by light which can pass through any considerable thickness of glass, the amount can hardly be within our means of ineasurement.

\section{Schumann Violet.}

In all the experiments hitherto described, the light traversed at least two centimetres of air and a quarter millimetre of quartz before reaching the gas. In order to see whether any effect was produced by rays of still shorter wave-length, such as air and quartz powerfully absorb, a different illumination vessel was used, made of brass and closed by a plate of fluorspar. The spark-gap was placed within two or three millimetres of the window, from which it was separated by a shield of fused quartz pierced with a small hole about the same diameter as the window; the condenser cylinder and rod were of graphite, and the sensitiveness of the electrometer was reduced to about one half. The following series of results was obtained :-

(1) Air alone at 5 litres per minate.................

(2) Air + about 10 per cent. chlorine, 5 litres per minute.

(3) Pure chlorine, 3 litres per minute ..............

(4) Air alone, 5 litres per minute..................

(5) Air +50 per cent. chlorine + Deflexions $\mathrm{cm}$.

$12,13 \quad 29,28$

$1,1-2,4,4$

0

12,14

29,20

(6) Pure chlorine, 3 litres per minute ..............

(7) Air alone, 5 litres per minute

$1121,17,16$

This was a long series of experiments, and the diminution in the effect observed with pure air towards the end of the series is easily explained by the fact that it is impossiblo to Phil. Mag. S. 6. Vol. 23. No. 137. May 1912. $3 \mathrm{E}$ 
keep the surface of the fluorspar perfectly free from a depnsit of aluminium given off by the sparks, despite brushing with a camel's-hair brush between each two successive illuminations.

The experiment is very conclusive in its evidence that the presence of chlorine destroys the conductivity produced in air, and that if pure chlorine itself is ionized it can only be so to a minute extent.

Effect of Moisture.-Carbon disulphide had been introduced into the earlier experiments to act as a conserving agent, but the experiments of Lenard had shown that traces of moisture were likely to act in a similar manner. In this connexion it is of great interest to remember that Baker found that the velocity of combination of hydrogen and chlorine exposed to bright light was very greatly dependent on the presence of a trace of moisture.

After passing over the usual drying apparatus and through the filter, the air was made to pass over a small surface of water, arranged by calibration to contribute about one gram of water vapour per cubic metre of air.

The results are tabulated below :-

Gas.

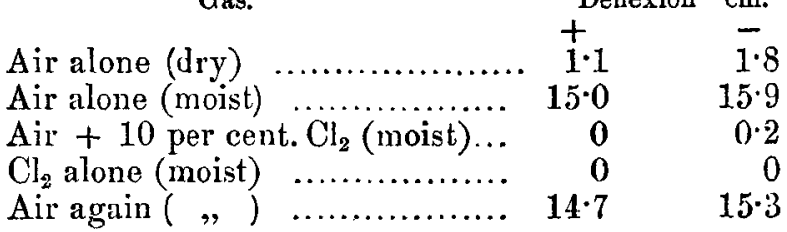

This table brings out two points very clearly.

(1) The presence of a trace of water vapour enormously increases the action upon air-in the case of the positive ions the increase is more than ten-fcld.

(2) Even this increased effect is completely obliterated by ehlorine.

Experiments with the Steam-Jet.

In conclusion it was a matter of interest and some importance to ascertain whether chlorine, in the various circuinstances under which it had been examined, gave rise to condensation nuclei or whether it prevented their formation. For this purpose the gases on leaving the illumination vessel were made to impinge against an orifice from which a jet of steam was issuing. ()n sparking for a few seconds the production of a cloud of steam showed instantly whether nuclei were present. 
The following table contains the result of the experiments, which were carried out in the sequence given.

Diam. of nuclei.

(1) Air alone without sparking.

(2) Air illuminated.

(3),$+\mathrm{CS}_{2}$.

(4), , $+\mathrm{CS}_{2}+\mathrm{Cl}_{2}$,

(5) " $+\mathrm{CS}_{2}+\mathrm{H}_{2} \mathrm{O}$.

(6) " $+\mathrm{CS}_{2}+\mathrm{H}_{2} \mathrm{O}+\mathrm{Cl}_{3}$.

(万) Pure $\mathrm{Cl}_{2}$.

(8) Air again.

(9) $"+\mathrm{Cl}_{2}(2)$.

(10) , $+\mathrm{CH}_{2}+\mathrm{CS}_{2}$.
A very feeble effect.

$\mathbf{m m}$.

Very considerable $6 \times 10^{-5}$ effect, grey.

Reddish brown $4 \times 10^{-6}$ cloud.

Scarcely any.

Powerful effect, $\quad 6 \times 10^{-6}$ grey,

Scarcely any.

Very slight.

Powerful effect, $\quad 6 \times 10^{-6}$ grey.

Scarcely any.

Scarcely any.

Thus it is seen that the results with the steam-jet fall into line exactly with those obtained by means of the electrometer, and indicate that chlorine forms neither ions nor nuclei, but that its presence is destructive of those otherwise produced in air. In this they confirm the experiments of Burgess and Chapman * as opposed to those of Bevan.

\section{Conclusions.}

The following effects have been confirmed or established:That under the influence of ultra-violet light,

(1) There is very little ionization produced, even in ordinary air, by light of wave-length longer than $200 \mu \mu$.

(2) That pure dry air is not greatly ionized by wave-lengths longer than $180 \mu \mu$, but with shorter vave-length the ionization is considerable.

(3) That the addition of a trace of water vapour to air enormously inereases the amount of ionization.

(4) That the addition of very small quantities of chlorine to ordinary air increases the amount of ionization, but that with more chlorine the reverse takes place.

(5) That pure chiorine is not ionized appreciably.

(6) That pure chlorine does not form condensation nuclei.

(7) That chlorine in considerable quantity destroys the ions and the nuclei otherwise formed in air.

* J, C. S. Trane. vol. xc, p. 1399 (1906). 
These results point to the fact that the atom of chlorine does not easily lose an electron, and this is what might be expected from its electronegative character. Affinity for negative electricity is the characteristic of electronegative elements. Hydrogen is a borderland element, taking up a position among the noble metals in virtue of its electrochemical properties ; it is distinctly more electropositive than copper, however, even at ordinary atmospheric pressure, and this is more marked at higher pressures. A possible explanation of the reaction between hydrogen and chlorine is that an atom of hydrogen, under the inflnence of ultra-violet light, emits an electron which immediately becomes attached to an atom of chlorine and chemical combination takes place *. There is some support for this hypothesis in the fact that the presence of hydrogen enormonsly increases the number of ions emitted by hot platinum and by the alkali metals at ordinary temperatures.

The fact that in many cases minute traces of foreign matter are requisite both for the ionization of gases and for their chemical combination is very striking; the two actions are probably either cause and effect or are attributable to some common cause. However this may be, it appears that actual liberation of electrons for any measureable period of time is not a necessary precursor of gaseous combination.

In conclusion I must express my indebtedness to Prof. Lenard for his kindness in allowing me to work in the Radiological Institute of the University of Heidelberg and for his guidance while the work was in progress. My thanks are also due to Prof. Becker and Dr. Ramsauer for kind assistance and advice.

Clifton College, Bristol.

LXXV. The Problem of the Weir. By J. H. C. SEARLE, B.Sc., Assistant Lecturer in Mathematics in the Victoria University, Manchester $\dagger$.

$\S 1$. THE problem of determining the motion of a stream 1 flowing in a horizontal channel, whose bed is subject to slight inequalities of level, was investigated by Lord Kelvin $\ddagger$, who assumed the flow to be irrotational, and obtained an approximate solution of the motion. Wien $\S$,

* Lenard and Ramsauer suggest that the light splits up the chlorine molecule into its two neutral atoms.

$\dagger$ Communicated by Prof. Horace Lamb, F.R.S.

t Phil. Mag. [5] vol. xxii. p. 3 ñ3 et seq.

$\S$ Wien, Hydrodynamik, p. 201. 